\title{
Relação entre saúde mental e vivência acadêmica dos estudantes de Engenharia de Alimentos e Engenharia Civil de uma universidade pública da região Nordeste
}

\author{
Relationship between mental health and academic experience of Food Engineering and Civil
}

Engineering students at a Public University in the Northeast region

Relación entre salud mental y experiencia académica de estudiantes de Ingeniería de Alimentos e

Ingeniería Civil em una Universidad Pública de la región Noreste

Recebido: 05/03/2021 | Revisado: 11/03/2021 | Aceito: 17/03/2021 | Publicado: 23/03/2021

\author{
Ivison Lima Dantas \\ ORCID: https://orcid.org/0000-0002-4222-7321 \\ Universidade Federal de Sergipe, Brasil \\ E-mail: ivisonlima007@ hotmail.com \\ Daise Lima Matos \\ ORCID: https://orcid.org/0000-0003-3769-9276 \\ Universidade Federal de Sergipe, Brasil \\ E-mail: deiselimamatos@ hotmail.com \\ Jane de Jesus da Silveira Moreira \\ ORCID: https://orcid.org/0000-0001-7586-4856 \\ Universidade Federal de Sergipe, Brasil \\ E-mail: jjsm@academico.ufs.br \\ Enaldo Vieira de Melo \\ ORCID: https://orcid.org/0000-0002-9314-4331 \\ Universidade Federal de Sergipe, Brasil \\ E-mail: evmsidarta@gmail.com \\ Edméa Fontes de Oliva Costa \\ ORCID: https://orcid.org/0000-0002-5577-0454 \\ Universidade Federal de Sergipe, Brasil \\ E-mail: edmeaolivacosta@gmail.com \\ Maria Terezinha Santos Leite Neta \\ ORCID: https://orcid.org/0000-0003-3982-910X \\ Universidade Federal de Sergipe, Brasil \\ E-mail: terezinhaleite@gmail.com
}

\begin{abstract}
Resumo
O objetivo deste estudo foi estimar a prevalência de transtornos mentais comuns (TMC) em graduandos dos cursos de Engenharia de Alimentos e Engenharia Civil de uma universidade pública da região Nordeste do Brasil e discutir sobre potenciais fatores associados. Foram utilizados dois questionários, um específico para traçar o perfil do aluno e o Self-Reporting Questionnaire (SRQ-20). A análise exploratória dos dados foi realizada mediante estatística descritiva e por meio do cálculo de razão de chances (odds ratio) simples e ajustado. No modelo final ajustado foram mantidas as variáveis independentes com associação ao desfecho após ajuste $(\mathrm{p} \leq 0,05)$, considerando-se o teste de verossimilhanças. Participaram do estudo $27,4 \%$ dos estudantes $(\mathrm{n}=161)$. A prevalência de TMC obtida para a população deste estudo foi alta $(73,91 \%)$. A análise de regressão logística mostrou forte associação dos indivíduos que não trabalham $(\mathrm{RC}=3,240)$, que consideram o curso menos do que esperavam $(\mathrm{RC}=2,682)$, cujo desempenho foi insatisfatório $(\mathrm{RC}=3,086)$ e que pensaram em desistir do curso $(\mathrm{RC}=3,613)$ com TMC. Esses resultados revelam a necessidade da discussão em torno desse tema e da adoção de medidas que possam evitar a evolução de TMC para quadros de transtornos mentais maiores, bem como buscar entender o que faz com que esses estudantes estejam insatisfeitos com o seu desempenho e com as estratégias de ensino e aprendizagem empregadas nesses cursos.
\end{abstract}

Palavras-chave: Ensino; Saúde mental; Universitários; Engenharia.

\begin{abstract}
This study was aimed at estimating the prevalence of Common Mental Disorders (CMD) in graduates of Food Engineering and Civil Engineering at a public university in the northeast region of Brazil and to discuss the potential associated factors. Two questionnaires were used, one for specifically profiling the student, as well as the SelfReporting Questionnaire (SRQ-20). The exploratory analysis of the data was carried out through descriptive statistics and through the calculation of simple and adjusted odd ratios. In the final adjusted model, independent variables correlated with the outcome after adjustment $(\mathrm{p} \leq 0.05)$ were maintained, considering the likelihood test, with $27.4 \%$ of
\end{abstract}


the students who participated in the study $(\mathrm{n}=161)$. The prevalence of CMD obtained for the population of this study was high $(73.91 \%)$. The logistic regression analysis showed a strong association of individuals with CMD who do not work $(\mathrm{RC}=3.240)$, who consider the course not as good as expected $(\mathrm{RC}=2.682)$, whose performance was unsatisfactory $(\mathrm{RC}=3.086)$ and who thought about giving up the course $(\mathrm{RC}=3.613)$. These results reveal the need for discussion on this topic and the adoption of measures that can prevent the evolution of CMD in patients with major mental disorders, as well as seeking to understand what makes these students dissatisfied with their performance and with their teaching and learning strategies employed in these courses.

Keywords: Teaching; Mental health; College students; Engineering.

\section{Resumen}

El objetivo del estudio fue estimar la prevalencia de Trastornos Mentales Comunes (TMC) en egresados de las carreras de Ingeniería en Alimentos e Ingeniería Civil de una universidad pública de la región noreste de Brasil y discutir los posibles factores asociados. Se utilizaron dos cuestionarios, uno específico para el perfil del alumno y el Cuestionario de Autorreporte (SRQ-20). El análisis exploratorio de los datos se realizó por medio de estadística descriptiva y con el cálculo de odds ratios simples y ajustadas. En el modelo final ajustado, las variables independientes se mantuvieron con asociación con el resultado después del ajuste $(\mathrm{p} \leq 0.05)$, considerando la prueba de verosimilitud. El estudio incluyó al $27,4 \%$ de los estudiantes $(\mathrm{n}=161)$. La prevalencia de TMC obtenida para la población de este estudio fue alta $(73,91 \%)$. El análisis de regresión logística mostró una fuerte asociación de individuos que no trabajan $(\mathrm{RC}=3.240)$, que consideran el curso menos de lo esperado $(\mathrm{RC}=2.682)$, cuyo desempeño fue insatisfactorio $(\mathrm{RC}=3.086)$ y que pensaron en renunciar al curso $(\mathrm{RC}=3.613)$ con $\mathrm{TMC}$. Estos resultados revelan la necesidad discutir este tema y adoptar medidas que puedan prevenir la evolución de TMC en pacientes con trastornos mentales importantes, así como buscar comprender lo qué hace que estos estudiantes estén insatisfechos con su desempeño y con las estrategias de enseñanza y aprendizaje empleadas en estos cursos.

Palabras clave: Docencia; Salud mental; Estudiantes universitarios; Ingeniería.

\section{Introdução}

Os transtornos mentais comuns (TMC) são caracterizados a partir de combinações de pensamentos, comportamentos anormais, sensações e emoções que influenciam diretamente na capacidade do indivíduo em executar simples atividades ao longo do dia ou até interferir em seu contato com outras pessoas (Opas, 2019).

Estudos epidemiológicos e de prevalência apontam, especialmente na comunidade acadêmica universitária, a presença desses transtornos em nível elevado quando comparado com adultos jovens não universitários e público geral (Eisenberg et al., 2007; Ibrahim et al., 2013).

Muitos desses estudos foram realizados com estudantes e profissionais da área da saúde em razão da vivência dessa população, uma vez que esses percebem e relatam com mais facilidade os sentimentos de sofrimento mental, sendo necessário aprender a viver com a angústia dos pacientes sem expor suas fragilidades (Quintana et al., 2004; Pereira et al., 2015). Pesquisas relacionadas a TMC na área de ciências exatas, especialmente nos cursos de engenharia, são raras e, por isso, faz-se necessário efetuar estudos que colaborem para o entendimento de fatores relacionados a essa população.

A pressão psicológica enfrentada pelos estudantes durante o curso, seja ela vivências e cobranças da própria faculdade ou expectativas familiares e pessoais, contribui para que sintomas de ansiedade e depressão sejam desencadeados, revelando-se através de traços psicológicos e fisiológicos (Sanches, 2012). Diante dessa realidade, as instituições de ensino superior estão experimentando taxas crescentes de transtornos mentais em seu público universitário (Auerbach et al., 2018).

Os cursos de engenharia, de modo geral, apresentam elevados índices de reprovação por disciplinas, o que desencadeia a desistência do aluno ou sua evasão para outro curso. Segundo dados institucionais (UFS, 2018, 2019), dentre os cursos de engenharia que apresentam elevado índice de desistência ou evasão de alunos, está o curso de Engenharia de Alimentos, que apresenta baixa taxa de sucesso $(13,71 \%)$. Em oposição, o curso de Engenharia Civil tem a taxa de sucesso $(65,16 \%)$ mais elevada entre as engenharias, com baixas taxas de evasão e abandono.

Nesse sentido, o objetivo deste estudo foi estimar a prevalência de TMC em graduandos dos cursos de Engenharia de Alimentos e Engenharia Civil de uma universidade pública da região Nordeste, com isso, buscando determinar os fatores associados e relacioná-los aos períodos de maior pressão psicológica dos cursos, enfrentados pelos discentes, em particular 
para o curso de Engenharia de Alimentos.

\section{Metodologia}

\section{População amostral}

Trata-se de um estudo transversal, realizado com os alunos dos cursos de graduação de Engenharia de Alimentos (agosto a setembro de 2019) e Engenharia Civil (janeiro a março de 2020) de uma universidade púbica da região Nordeste. Todos os alunos ativos do curso de Engenharia de Alimentos (População = 187) e Engenharia Civil (População = 400) foram convidados a participar deste estudo.

A aplicação dos questionários, específico e SRQ-20, foi efetuada após aprovação do comitê de ética com número do Parecer 3.377.830. Buscou-se motivar e cativar os alunos a participarem da pesquisa, para tanto, ressaltando-se a importância desta para os cursos mencionados. Ambos os questionários foram aplicados utilizando-se o formulário Google (Google forms), disponibilizados via link aos participantes.

Os sujeitos receberam o Termo de Consentimento Livre e Esclarecido (TCLE), por meio do qual foram informados sobre a finalidade da pesquisa, reservando-lhes o direito de aceitar ou não participar desta. Aceitaram participar do estudo 161 estudantes, sendo 89 pertencentes ao curso de Engenharia Civil e 72, ao de Engenharia de Alimentos.

\section{Coleta de dados}

\section{Questionário específico}

Este é um questionário autoaplicável com 28 questões, sendo 2 abertas e 26 fechadas, pré-codificadas, relativas às características sociodemográficas, ao processo de ensino e aprendizagem, aos aspectos pessoais ligados à escolha da profissão e às experiências psicoemocionais recentes.

Neste estudo, a associação da variável dependente (TMC) com as variáveis explicativas (sexo, idade, estado civil, período atual, período de maior dificuldade, atividade física, religião, procedência, outra ocupação, satisfação com a escolha profissional, sentimento em relação ao trabalho, desempenho profissional, satisfação com as estratégias de ensino, sentimento em relação às atividades da faculdade, presença de doença física, presença de doença mental prévia, automedicação, autoavaliação de tensão emocional, horas de lazer, atividade física, apoio emocional, expectativas para o futuro, sentimento de felicidade) foi investigada e analisada por meio do cálculo de razão de chances (odds ratio) simples e ajustado.

\section{Self-Reporting Questionnaire (SRQ-20)}

O SRQ-20 é um questionário que contém 20 questões em sua estrutura e foi construído com o intuito de analisar a presença de traços ansiosos, depressivos e somatoformes em indivíduos, introduzido por Harding et al. (1980), sendo usado pela Organização Mundial de Saúde (OMS) para "screening" de TMC em populações em atenção primária. Este instrumento foi traduzido para diferentes idiomas, sendo validado no Brasil, por Mari e Williams (1986).

O SRQ-20 é uma ferramenta autoaplicável. Esse instrumento possui 20 questões com respostas binárias que possibilitam o estabelecimento de um escore. O ponto de corte mais adequado do SRQ-20 para o estudo em questão foi determinado a partir do trabalho original de Mari e Williams (1985) e de outros estudos (Ludermir \& Lewis, 2005; Costa et al., 2010; Grether, Becker \& Menezes, 2019; Lima \& Brito, 2020) como sendo, nos homens, $\leq 5$ para os casos não suspeitos e $\geq 6$ para os casos suspeitos, enquanto, nas mulheres, $\leq 7$ para os casos não suspeitos e $\geq 8$ para os casos suspeitos.

\section{Compilação dos dados e Testes estatísticos}

Os dados do estudo foram tabulados em uma planilha eletrônica do programa "Excel for Windows" em forma de 
banco de dados eletrônico. Todos os testes estatísticos foram executados utilizando o programa SPSS Statistics IBM (versão de avaliação). A prevalência de TMC e as variáveis associadas foram identificadas de acordo com as seguintes etapas:

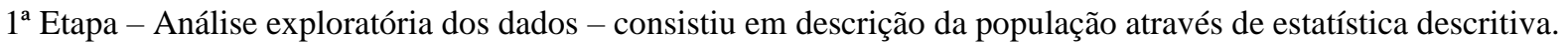

$2^{\mathrm{a}}$ Etapa - Análise simples - elaboração de tabelas, cálculo de razões de chances e seus respectivos intervalos de 95\% de confiança, análise estratificada e cálculo de razões de chances ajustados pelo método de Mantel e Haenszel (Daniel, 1995).

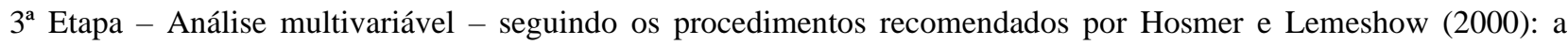
análise multivariada foi executada por meio da regressão logística ("backstepwise"). Foram inclusas no modelo as variáveis que mostraram associação com desfecho com $p \leq 0,25$. Para o modelo final ajustado foram mantidas as variáveis independentes com associação ao desfecho após ajuste ( $\mathrm{p} \leq 0,05)$, considerando-se o teste de verossimilhanças ("likelihoodratiotest").

\section{Resultados}

O presente estudo contou com a participação de $27,4 \%$ dos estudantes $(n=161)$, sendo $44,7 \%$ desses do curso de graduação de Engenharia de Alimentos e 55,3\% de Engenharia Civil. A idade média dos alunos era de 22,6 $\pm 3,9$ anos e o estado civil da maioria (91,3\%), solteiro. Dos participantes, 58,4\% pertenciam ao sexo feminino. Grande parte desses indivíduos era da capital Aracaju (39,8\%) e do interior do estado (37,9\%), conforme apresentado na Tabela 1.

Tabela 1. Distribuição dos alunos segundo os dados de variáveis sociodemográficas e os aspectos pessoais.

\begin{tabular}{|c|c|c|}
\hline Variáveis & $N=161$ & $\%$ \\
\hline Idade média em anos & $22,6 \pm 3,9$ & \\
\hline \multicolumn{3}{|l|}{ Curso } \\
\hline Engenharia Civil & 89 & 55,3 \\
\hline Engenharia de Alimentos & 72 & 44,7 \\
\hline \multicolumn{3}{|l|}{ Sexo } \\
\hline Feminino & 94 & 58,4 \\
\hline Masculino & 67 & 41,6 \\
\hline \multicolumn{3}{|l|}{ Estado civil } \\
\hline Solteiro & 147 & 91,3 \\
\hline Casado ou separado/divorciado & 14 & 8,7 \\
\hline \multicolumn{3}{|l|}{ Procedência } \\
\hline Capital (Aracaju) & 64 & 39,8 \\
\hline Interior de Sergipe & 61 & 37,9 \\
\hline Outros estados ou exterior & 36 & 22,3 \\
\hline Praticante de alguma religião & 93 & 57,8 \\
\hline Atividade física & 82 & 50,9 \\
\hline \multicolumn{3}{|l|}{ Reside com } \\
\hline Familiares & 112 & 69,6 \\
\hline Amigos/colegas & 34 & 21,1 \\
\hline Sozinho & 15 & 9,3 \\
\hline Tem familiar engenheiro & 41 & 25,5 \\
\hline Trabalha, além de estudar & 53 & 32,9 \\
\hline
\end{tabular}

Fonte: Autores (2021).

É possível ainda constatar, conforme a Tabela 1, que muitos dos alunos residiam com seus familiares $(69,6 \%)$ e apenas 32,9\% deles conciliavam trabalho e estudo. A maioria (74,5\%) não tinha membros da família cuja profissão fosse a engenheira. Dos estudantes, 57,8\% afirmaram seguir alguma religião e 50,9\%, praticar algum tipo de atividade física. $\mathrm{Na}$ 
Tabela 2, expõem-se os resultados relacionados aos aspectos de ensino e aprendizagem.

Tabela 2. Caracterização dos alunos segundo variáveis do processo ensino e aprendizagem.

\begin{tabular}{|c|c|c|}
\hline Variáveis & $N=161$ & $\%$ \\
\hline \multicolumn{3}{|l|}{ Momento atual no curso } \\
\hline $1^{\circ}$ e $2^{\circ}$ anos do curso & 43 & 26,7 \\
\hline $3^{\circ}$ e $4^{\circ}$ anos do curso & 66 & 41,0 \\
\hline $5^{\circ}$ ano do curso & 17 & 10,6 \\
\hline Mais de $5^{\circ}$ anos & 35 & 21,7 \\
\hline \multicolumn{3}{|l|}{ Período de maior dificuldade } \\
\hline $1^{\circ}$ período & 32 & 19,9 \\
\hline $2^{\circ}$ período & 23 & 14,3 \\
\hline $3^{\circ}$ período & 43 & 26,1 \\
\hline $4^{\circ}$ período & 24 & 14,9 \\
\hline $5^{\circ}$ período & 14 & 8,7 \\
\hline $6^{\circ}$ período & 11 & 6,8 \\
\hline $7^{\circ}$ período & 5 & 3,1 \\
\hline $8^{\circ}$ período & 1 & 0,6 \\
\hline $9^{\circ}$ período & 6 & 3,7 \\
\hline $10^{\circ}$ período & 3 & 1,9 \\
\hline Satisfeito com a carreira de engenheiro & 123 & 76,4 \\
\hline Teve reprovação ao longo do curso & 132 & 82,0 \\
\hline \multicolumn{3}{|l|}{ Sensação em relação ao curso } \\
\hline É menos do que eu esperava & 55 & 34,2 \\
\hline É o que eu esperava ou mais & 106 & 65,9 \\
\hline \multicolumn{3}{|l|}{ Quantos anos levou para entrar neste curso de engenharia } \\
\hline 1 ano & 138 & 85,7 \\
\hline 2 anos & 16 & 9,9 \\
\hline 3 anos ou mais & 7 & 4,3 \\
\hline Pensou em abandonar o curso & 103 & 64,0 \\
\hline \multicolumn{3}{|l|}{ Desempenho no curso } \\
\hline Satisfatório ou regular & 112 & 69,6 \\
\hline Insatisfatório & 49 & 30,4 \\
\hline $\begin{array}{l}\text { Satisfeito com as estratégias de ensino e aprendizagem } \\
\text { usadas em seu curso de engenharia }\end{array}$ & 64 & 39,8 \\
\hline Apoio emocional durante o curso & 34 & 21,1 \\
\hline
\end{tabular}

Fonte: Autores (2021).

Como identificado na Tabela 2, a maioria dos discentes $(85,7 \%)$ levou um ano para ingressar em seu curso de engenharia. Muitos desses $(41,0 \%)$ encontravam-se entre o terceiro e quarto ano do curso. É válido expor que um número significativo de estudantes $(21,7 \%)$ estava a mais de cinco anos no curso. Os períodos de maiores dificuldades mencionados compreenderam a primeira metade do curso, com ênfase para o primeiro $(19,9 \%)$ e terceiro períodos $(26,1 \%)$.

Dos participantes, $82 \%$ reprovaram em alguma disciplina ao longo do curso, 64\% pensaram abandoná-lo e 69,6\% consideraram seu desempenho satisfatório ou regular. Além do mais, 76,4\% estavam satisfeitos com a escolha de carreira, $39,8 \%$, satisfeitos com as estratégias de ensino e aprendizagem usadas em seu curso, $21,1 \%$ referiram receber apoio emocional e 65,9\% afirmaram que o curso era o que esperavam ou mais. Na Tabela 3 é possível verificar os fatores associados às variáveis de transtornos mentais comuns. 
Tabela 3. Distribuição dos fatores associados, entre os estudantes de engenharia, com transtornos metais comuns.

\begin{tabular}{|c|c|c|c|}
\hline \multirow[t]{2}{*}{ Variáveis } & \multicolumn{2}{|c|}{ COM TMC } & \multirow[t]{2}{*}{$p$} \\
\hline & $\mathbf{N}=\mathbf{1 1 9}$ & $73,9(\%)$ & \\
\hline Curso & & & 0,94 \\
\hline Engenharia Civil & 66 & 55,5 & \\
\hline Engenharia de Alimentos & 53 & 44,5 & \\
\hline Sexo & & & 0,1 \\
\hline Feminino & 74 & 62,2 & \\
\hline Masculino & 45 & 37,8 & \\
\hline Prática atividade física & 58 & 48,7 & 0,349 \\
\hline Praticante de alguma religião & 63 & 52,9 & 0,037 \\
\hline Tem familiares engenheiro & 32 & 26,9 & 0,485 \\
\hline Trabalha, além de estudar & 33 & 27,7 & 0,018 \\
\hline Satisfeito com a carreira & 82 & 68,9 & 0,0001 \\
\hline Teve reprovação ao longo do curso & 100 & 84,0 & 0,255 \\
\hline Sensação em relação ao curso & & & 0,018 \\
\hline É menos do que eu esperava & 48 & 40,3 & \\
\hline É o que eu esperava ou mais & 71 & 59,7 & \\
\hline Pensou em abandonar o curso & 87 & 73,1 & 0,0001 \\
\hline Desempenho no curso & & & 0,010 \\
\hline Satisfatório ou regular & 75 & 63,0 & \\
\hline Insatisfatório & 44 & 37,0 & \\
\hline $\begin{array}{l}\text { Satisfeito com as estratégias de ensino e aprendizagem } \\
\text { usadas em seu curso de engenharia }\end{array}$ & 42 & 35,3 & 0,052 \\
\hline Apoio emocional durante o curso & 20 & 16,8 & 0,024 \\
\hline Apresenta ou apresentou algum transtorno mental & 19 & 16,0 & 0,006 \\
\hline Fez ou faz uso de algum psicofármaco & 20 & 16,8 & 0,005 \\
\hline Fez ou faz uso de alguma substância psicoativa lícita & 23 & 19,3 & 0,008 \\
\hline Fez ou faz uso de alguma substância psicoativa ilícita & 13 & 10,9 & 0,091 \\
\hline $\begin{array}{l}\text { Diante de situações de conflito nas relações } \\
\text { interpessoais }\end{array}$ & & & 0,030 \\
\hline Retrai-se ou reage agressivamente & 64 & 55,8 & \\
\hline Dialoga & 55 & 46,2 & \\
\hline $\begin{array}{l}\text { Passou por alguma experiência com doença grave em } \\
\text { si ou na família }\end{array}$ & 73 & 61,3 & 0,122 \\
\hline
\end{tabular}

$p \leq 0,5$ apresenta diferença significativa. Fonte: Autores (2021).

De acordo com a nota de corte ponderada para essa pesquisa, 73,91\% dos participantes apresentavam TMC. Não houve diferença significativa relacionada à presença ou ausência de TMC no quesito idade. Como observado na Tabela 3, dos indivíduos que assinalavam TMC, 44,5\% eram do curso de Engenharia de Alimentos e 55,5\%, de Engenharia Civil. A presença de TMC encontrada para o sexo feminino $(62,2 \%)$ foi alta. Da população que praticava alguma atividade física, 48,7\% revelaram TMC e 52,9\% dos que seguiam alguma religião indicavam tal ocorrência.

Dos estudantes que tinham familiar engenheiro, 26,9\% eram casos de TMC e daqueles que trabalhavam além de estudar, 27,7\%. Quanto aos alunos satisfeitos com a escolha de carreira, 68,9\% deles eram casos de TMC, ao ponto que, das 
pessoas que reprovaram em alguma disciplina ao longo do curso, 84,0\% dessas indicavam tal ocorrência. Em relação aos discentes que consideravam o curso ser o que esperavam ou mais, $59,7 \%$ eram casos de TMC.

A respeito dos indivíduos que pensaram em abandonar o curso, 73,1\% apresentavam TMC, ao passo que 63,0\% dos que consideravam o seu desempenho satisfatório ou regular eram casos desse evento. Além disso, 35,3\% dos alunos satisfeitos com as estratégias de ensino e aprendizagem utilizadas em seu curso de engenharia, revelavam TMC e 55,8\% daqueles que diante de situações de conflitos nas relações interpessoais se retraem ou reagem agressivamente, também, eram casos de TMC.

Dos estudantes que afirmaram receber apoio emocional durante o curso, 16,8\% eram casos de TMC, ao mesmo tempo que $16,0 \%$ dos que apresentavam ou apresentaram algum transtorno mental, 16,8\% que fizeram ou faziam uso de algum psicofármaco, 19,3\% que fizeram ou faziam uso de alguma substância psicoativa lícita e 10,9\% que fizeram ou faziam uso de alguma substância psicoativa ilícita estavam dentro do grupo com prevalência de TMC. Dentre os indivíduos que passaram por alguma experiência com doença grave, em si ou na família, a prevalência de TMC foi de 61,3\%. A partir dos dados de associação a TMC foram determinadas as razões de chance e os resultados expressos na Tabela 4.

Tabela 4. Razões de chance (Odss ratio) para variáveis relacionadas e transtornos metais comuns em estudantes de engenharia.

\begin{tabular}{lcc}
\hline \multicolumn{1}{c}{ Variáveis } & RC & IC 95\% \\
\hline Sexo & 1,809 & $0,889-3,679$ \\
\hline Estado Civil & 2,312 & $0,752-7,111$ \\
\hline $\begin{array}{l}\text { Insatisfeito com as estratégias de ensino e aprendizagem } \\
\text { usadas em seu curso de engenharia }\end{array}$ & 18,500 & $2,451-139,646$ \\
\hline $\begin{array}{l}\text { Sensação em relação ao curso (é menos do que } \\
\text { esperava) }\end{array}$ & 3,380 & 0,102 \\
\hline Desempenho no curso (insatisfatório) & & 0,005 \\
\hline $\begin{array}{l}\text { Diante de situações de conflito nas relações } \\
\text { interpessoais (retrai-se ou reage agressivamente) }\end{array}$ & 4,341 & $1,388-8,234$ \\
\hline Pensou em abandonar o curso & 2,596 & 0,007 \\
\hline Não trabalha, além de estudar & $4,418-11,864$ \\
\hline Apoio emocional durante o curso & $2,369-5,478$ \\
\hline Teve reprovação ao longo do curso & 2,475 & 0,004 \\
\hline Insatisfeito com a carreira & 1,645 & 0,012 \\
\hline $\begin{array}{l}\text { Passou por alguma experiência com doença grave em si } \\
\text { ou na família }\end{array}$ & 2,017 & $1,146-4,899$ \\
\hline
\end{tabular}

RC: razão de chance; IC: intervalo de confiança; $\mathrm{p} \leq 0,25$ apresenta diferença significativa. Fonte: Autores (2021).

Na observação feita para a Tabela 4, no que tange às variáveis associadas ao processo de ensino e aprendizagem, apresentavam maior relação com o surgimento de TMC os estudantes que reprovaram em alguma disciplina $(\mathrm{RC}=1,645)$, que pensaram em abandonar o curso $(\mathrm{RC}=4,418)$, que estavam insatisfeitos com a escolha de carreira $(\mathrm{RC}=2,017)$, com seu desempenho $(\mathrm{RC}=4,341)$, com as estratégias de ensino e aprendizagem usadas em seu curso de engenharia $(\mathrm{RC}=18,500)$ e que consideravam que o curso era menos do que esperavam $(\mathrm{RC}=3,380)$.

Quanto às variáveis relacionadas aos aspectos pessoais e emocionais dos alunos, a chance de desenvolver TMC foi maior entre estudantes do sexo feminino $(\mathrm{RC}=1,809)$, que não trabalhavam $(\mathrm{RC}=2,369)$, que passaram por alguma experiência de doença grave em si ou na família $(\mathrm{RC}=1,746)$, que diante de situações de conflito nas relações interpessoais se retraem ou reagem agressivamente $(\mathrm{RC}=2,596)$, que consideravam não receber apoio emocional no curso $(\mathrm{RC}=2,475)$ e cujo estado civil era solteiro $(\mathrm{RC}=2,312)$. 
A partir dos valores de RC cujo $p$ foi significativo $(p<0,05)$, foram selecionadas as variáveis para o ajuste na análise de regressão. Foram inseridas as variáveis sexo, estado civil, insatisfação com as estratégias utilizadas em seu curso de engenharia, sensação em relação ao curso, desempenho no curso de engenharia, situações de conflito nas relações interpessoais, não trabalha (além de estudar), satisfação com a escolha de carreira, doença grave em si ou na família, já pensou em abandonar o curso, apoio emocional durante o curso e reprovação. O modelo final ajustado foi obtido considerando as variáveis que, após o teste de verossimilhança, permaneceram no modelo, conforme exposto na Tabela 5.

Tabela 5. Modelo ajustado de regressão logística para variáveis relacionadas e transtornos metais comuns em estudantes de engenharia.

\begin{tabular}{lccc}
\hline \multicolumn{1}{c}{ Variáveis } & RC & Intervalo (95\%) & $\boldsymbol{p}$ \\
\hline Não trabalha (além de estudar) & 3,240 & $1,410-7,445$ & 0,006 \\
\hline $\begin{array}{l}\text { Sensação em relação ao curso (é menos do que } \\
\text { esperava) }\end{array}$ & 2,682 & $1,023-7,032$ & 0,045 \\
\hline Desempenho no curso (insatisfatório) & 3,086 & $1,043-9,132$ & 0,042 \\
\hline Pensou em abandonar o curso & 3,613 & $1,601-8,154$ & 0,002 \\
\hline
\end{tabular}

RC: razão de chance; IC: intervalo de confiança; $p \leq 0,05$ apresenta diferença significativa. Fonte: Autores (2021).

Das variáveis associadas aos TMC que foram inseridas para o ajuste do modelo, permaneceram após a análise de regressão as variáveis não trabalha (além de estudar), sensação em relação ao curso (é menos do que esperava), desempenho no curso (insatisfatório) e pensou em abandonar o curso, como é visto na Tabela 5. Esse modelo descreve comportamento semelhante para os discentes nos quatro grupos.

Mediante o resultado do ajuste foi observada a forte associação dos indivíduos que não trabalhavam $(\mathrm{RC}=3,240)$, que consideravam o curso menos do que esperavam $(\mathrm{RC}=2,682)$, cujo desempenho foi insatisfatório $(\mathrm{RC}=3,086)$ e que pensaram em abandonar o curso $(\mathrm{RC}=3,613)$ com TMC.

\section{Discussão}

A prevalência $(73,91 \%)$ de TMC obtida para a população deste estudo foi alta e superior às prevalências observadas em estudos brasileiros envolvendo universitários de diferentes regiões e áreas do conhecimento, variando de 18,5\% a 60,0\% (Ludemir \& Melo Filho, 2002; Facundes \& Ludermir, 2005; Costa et al., 2010; Moreira et al., 2011; Silva \& Neto, 2014; Junior et al., 2015; Perini, Delanogare \& Souza, 2019; Lima \& Brito, 2020).

Para Mojtabai et al. (2015) e Auerbach et al. (2016), a alta prevalência de TMC em estudantes universitários vem crescendo cada vez mais. O desgaste emocional sofrido por parte desse público dificulta o gerenciamento de suas atividades ao longo do dia, causando assim prejuízos ao desempenho acadêmico e pessoal.

A alta prevalência de TMC no público feminino da pesquisa revela que as mulheres são mais suscetíveis a desordens emocionais, pois essas desempenham dupla função, necessitando dar conta dos estudos e ainda ajudar nas atividades da casa, além de naturalmente terem de lidar com questões hormonais, aspecto natural à realidade feminina, o que pode gerar repercussões importantes à sua saúde devido ao desgaste físico e mental (Santos et al., 2017). Marin-Leon et al. (2007) e Silva et al. (2019) apontam que esse resultado é esperado para as mulheres, pois elas têm maior prevalência de transtornos mentais.

Em países desenvolvidos, os TMC são o terceiro problema de saúde entre as mulheres; e, nos países subdesenvolvidos, é o quinto problema nesse grupo (Ludemir \&Filho, 2002). Marin-Leon et al. (2007) e Moreira et al. (2011) indicam que a prevalência ou diagnóstico de TMC em mulheres é maior que nos homens, visto que as mulheres têm mais facilidade em relatar suas emoções e procuram ajuda profissional. Todavia o público do sexo feminino $(58,4 \%)$, neste estudo, 
foi levemente maior que o masculino (41,6\%), isso consequentemente expõe a presença maior de TMC nas mulheres.

Conquanto a associação de TMC tenha sido alta para os acadêmicos dos cursos analisados, o número de indivíduos que tiveram diagnóstico médico comprovado e que fizeram ou faziam o uso de psicofármaco ou substâncias psicoativas lícitas foi baixo. Fato semelhante é registrado em um trabalho desenvolvido com graduandos do curso de Enfermagem (Silva et al., 2019).

A presente pesquisa revelou que muitos dos estudantes vivenciaram experiência com doença grave em si ou na família. Isso pode desencadear uma instabilidade emocional no aluno e, consequentemente, ocasionar uma diminuição em seu rendimento acadêmico a curto e longo prazo (Soares \& Collares, 2006). Muitas pessoas têm almejado melhor qualidade de vida; nesse sentido, uma simples atividade descontraída colabora para o seu bem-estar físico, mental e social.

A prática de atividade física, junto com o lazer, favorece positivamente a saúde das pessoas (Del Duca et al., 2014). O presente estudo indica que existe uma leve tendência no aumento de TMC quando os indivíduos não praticam atividade física. Holley et al. (2011), Lincoln, et al. (2011) e Wipfli et al. (2011) destacam a eficácia da utilização dessa prática enquanto terapia não medicamentosa para a saúde mental de pessoas que possuem algum transtorno.

Apesar da prevalência de TMC no público analisado ser alta, a maioria dos estudantes encontrava-se satisfeita com a sua escolha de carreira. No entanto, os alunos que reprovaram, pensaram em abandonar o curso e consideraram esse ser menos do que o esperado, apresentando forte associação com TMC, pois a chance do estudante que considera o seu desempenho insatisfatório largar o curso é maior, já que, certamente, o indivíduo será influenciado pela análise do seu desempenho, fatores externos e internos a ele (Oikawa, 2019).

Embora a maioria dos indivíduos pesquisados estivesse satisfeita com a escolha da carreira de engenheiro, foi possível observar que, mesmo aqueles que não tiveram reprovações em disciplinas, apresentavam prevalência de TMC. A autocobrança excessiva em alcançar sempre o sucesso desencadeia sentimentos que afetam a psique e isso os deixa suscetíveis a uma desordem psíquica (Gomes \& Calixto, 2019; Leão et al., 2018).

A falta de apoio emocional relatada pelos participantes desta pesquisa corrobora o resultado elevado de TMC. A ausência desse apoio por parte da universidade aos alunos é algo que pode refletir em sua evasão do curso, pois, em alguns momentos, o aluno pode não ser capaz de lidar com situações provindas da realidade universitária. Entretanto é preciso compreender todos os fatores que podem estar associados a isso (Ogushi \& Bardagi, 2015). A presença de profissionais especializados (psicólogos) nos campi universitários muitas vezes é pequena, o que impossibilita a assistência a todos os alunos, visto que o número de matriculados, no geral, é superior ao número de profissionais da área de saúde mental (Bardagi \& Hutz, 2005).

Os períodos que compreendem a primeira metade dos cursos foram apontados como aqueles de maiores dificuldades e pressões psicológicas, com destaque para o primeiro e terceiro períodos da matriz curricular de ambos os cursos. Isso é um fato bastante interessante, pois, das disciplinas ofertadas nos períodos mencionados, três são ofertadas igualmente a ambos os cursos no primeiro período e quatro no terceiro período, como apresentado no projeto político pedagógico de cada curso, segundo a Resolução n. ${ }^{\circ}$ 02/2007/Conepe e Resolução n. ${ }^{\circ}$ 174/2009/Conepe. Essas semelhanças de disciplinas ocorrem porque os cursos de engenharia apresentam bases curriculares similares nos primeiros dois anos e meio de graduação.

O contato inicial do estudante com a universidade é crucial, visto que sua saída do Ensino Médio para o Ensino Superior, falta de maturidade, chegada da fase adulta, assim como alterações nas relações familiares, pessoais e afetivas interferem diretamente na adaptação, vivência e realidade do estudante na universidade. Por isso, os indivíduos tendem a considerar o primeiro período como um dos mais difíceis, pois estão vivendo o período de transição de um sistema para outro e a sua adaptação à fase adulta, o que afeta positiva ou negativamente o seu psicológico (Polydoro et al., 2001; Arnett, 2007).

Na percepção dos alunos da Engenharia de Alimentos, no terceiro período, junto com as demais disciplinas ofertadas 
nele, a matéria de Termodinâmica Aplicada se apresenta com elevado grau de dificuldade, situação que pode contribuir para o agravamento emocional dos estudantes, evidenciando que aspectos emocionais do indivíduo estão atrelados às disciplinas vivenciadas ao longo do curso (Rêgo \& Rocha, 2009).

A insatisfação dos alunos com as estratégias de ensino e aprendizagem aplicadas nos cursos revelou-se elevada. Essa é uma ocorrência intrigante, pois, apesar de insatisfeitos com tais estratégias, de acordo com os dados institucionais (UFS, 2018, 2019), os alunos da Engenharia Civil, embora apresentem maior prevalência de TMC, possuem os melhores índices acadêmicos, sendo isso comprovado pelas taxas de sucesso elencadas ao curso, ao ponto que a situação dos alunos da Engenharia de Alimentos é completamente oposta. As estratégias usadas pelo professor em sala de aula influenciam diretamente no aprendizado do aluno e em sua satisfação ou não quanto a esse aspecto (Costa, 2011).

O modelo de análise de regressão descreve o mesmo perfil de resposta para os estudantes que consideravam insatisfatório o seu desempenho, o curso menos do que esperavam, pensaram em abandonar o curso e que não trabalham, além de estudar.

A prevalência de TMC entre os indivíduos que consideravam insatisfatório o seu desempenho e o curso menos do que o esperado se relaciona entre si, uma vez que, se o curso estiver abaixo do que foi projetado pelo aluno, isso gera um sentimento de frustração, o que afeta diretamente o seu rendimento acadêmico e impulsiona-o a abandonar o curso, ao ponto que isso acarreta em possíveis reações fisiológicas, emocionais, comportamentais e cognitivas no indivíduo, sentimentos esses que estão interligados aos parâmetros avaliados (Bohry, 2007; Tosevski et al., 2010; Carvalho et al., 2015; Certo, 2016).

A chance de desenvolver TMC é duas vezes maior nos alunos que consideravam o curso ser menos do que o esperado ( $\mathrm{RC}=2,682)$ e três vezes maior para os que consideravam seu desempenho insatisfatório $(\mathrm{RC}=3,086)$ e que pensaram em abandonar o curso $(\mathrm{RC}=3,613)$. Esses sentimentos podem estar atrelados à grade curricular, carga horária, ter ingressado no curso por acaso, não adaptação à realidade do curso, défice proveniente da educação básica, falta de maturidade, reprovações consecutivas em disciplinas, ausência de diálogo com professores, renda, problemas familiares ou pessoais, entre outros fatores que podem contribuir de forma positiva ou negativa para a continuidade do aluno no curso e o desencadeamento dos transtornos metais (Fiedler, 2008; Almeida \& Godoy, 2016; Freitas et al., 2017).

A prevalência de TMC evidenciada para os estudantes que não trabalhavam foi três vezes ( $\mathrm{RC}=3,240$ ) maior do que para os indivíduos que exerciam alguma atividade profissional. De acordo com Sampson et al. (2013) e Silva e Andrade (2016), os indivíduos que têm mais tempo livre são suscetíveis a pensamentos que os fazem sofrer.

A maturidade do indivíduo é um fator a ser considerado, visto que aqueles que trabalham amadurecem mais rapidamente em função das exigências e maiores responsabilidades assumidas, assim, passam a ter um olhar diferenciado sobre a sua formação profissional, fazendo com que entendam e sofram menos com as exigências da formação profissional (Gondim, 2002; Cosme, 2017). Entretanto é comprovado que uma carga de trabalho superior a 20 horas por semana para pessoas que estudam tem impacto negativo, posto que elas ficam limitadas quanto às horas de estudo fora da sala de aula e, consequentemente, podem ter dificuldades em se envolver com experiências que poderiam auxiliar na obtenção de melhores resultados acadêmicos (Logan, Hughes \& Logan, 2016).

Uma pesquisa indiana realizada com estudantes de engenharia demonstra que o desempenho acadêmico é influenciado pelos fatores promotores de estresse (Manimaran, Jayakumar \& Lakshmi, 2016). Os resultados encontrados no presente estudo evidenciaram que as situações provindas do cotidiano da universidade provocavam sofrimento mental nos alunos de graduação do curso de Engenharia de Alimentos e Engenharia Civil, o que foi comprovado pela alta prevalência de TMC no público avaliado.

Esse estudo apresenta limitações relativas ao período de aplicação dos questionários que foram em momentos diferentes e pelo $\mathrm{N}$ amostral por curso, considerado pequeno, o que pode influenciar a generalização dos resultados para os 
cursos avaliados, além de se tratar de um estudo transversal descritivo analítico e não poder inferir causalidade. Entretanto as observações feitas ao longo deste trabalho contribuem de forma significativa para a compreensão dos fatores associados à geração de TMC, possibilitando a adoção de medidas preventivas que venham a minimizar o sofrimento psíquico dos estudantes, assim, evitando a sua evolução para quadros de transtornos mentais maiores, como a depressão. Ademais, este é um estudo inédito que aborda especificamente dois cursos de engenharia, traçando um comparativo entre eles com relação à presença de TMC e seus fatores associados.

Acredita-se que a divulgação do presente estudo de modo amplo à toda a comunidade acadêmica pesquisada, possa contribuir para uma reflexão profunda de discentes, docentes e gestores acerca do seu papel na promoção, manutenção e prevenção dos dados encontrados. Dessa forma, pensando na prevenção sugere-se que os Núcleos Docente Estruturantes, de ambos os cursos, ofertem capacitação docente frequente de modo a promover cada vez mais a percepção destes acerca da identificação de problemas sobre o tema em estudo.

Entende-se que uma maior aproximação dos gestores aos serviços oferecidos pela Clínica de Psicologia da Assistência Estudantil, vinculada a PROEST (Pró-Reitoria de Assuntos Estudantis), é indispensável visando promover atividades de relaxamento, técnicas de "mindfulness" e atendimento individualizado aos alunos e professores. Como forma de reforçar as ações anteriores, sugere-se o encaminhamento destes aos serviços de psicologia e de psiquiatria do Hospital Universitário sempre que se fizer necessário.

\section{Conclusão}

A alta prevalência de TMC entre os estudantes de Engenharia de Alimentos e Engenharia Civil do presente estudo, relacionados aos fatores de risco voltados aos aspectos pessoais, psicoemocionais e ao processo de ensino e aprendizagem, revelaram a necessidade urgente de uma atenção específica para questões voltadas ao ensino e à aprendizagem empregadas nesses cursos e a adoção de medidas para o acompanhamento da saúde mental desses estudantes.

É relevante frisar que este estudo foi desenvolvido para entender se a prevalência dos TMC no curso de Engenharia de Alimentos poderia ser um fator relacionado ao alto índice de desistência e insucesso por parte dos alunos nesse curso, como revelam os dados institucionais. Entretanto, ao contrário do que se esperava, foi possível verificar que a prevalência de TMC é superior nos alunos do curso de Engenharia Civil, que apresentam dados de sucesso opostos aos da Engenharia de Alimentos. Logo, o fato de os discentes apresentarem TMC não é um fator definitivo para a taxa de sucesso do curso.

Outros estudos devem ser realizados visando responder quais os fatores estão, especificamente, voltados para a questão do processo de ensino e aprendizagem utilizados nesses cursos e que geram insatisfação por parte dos alunos, assim como compreender em qual momento surge essa insatisfação e os possíveis motivos que contribuem para tal fato suceder.

\section{Agradecimentos}

À Deus por em todos os momentos ter conduzido o nosso trabalho. As dificuldades existiram, porém, Ele sempre nos concedeu a força, direção, sabedoria e discernimentos necessários para agir diante a todas as situações. Certamente, sem a ação do Espírito Santo em nós, nada seria possível.

Depois, agradecemos a todos que se empenharam na realização desse estudo (alunos, professores, colegas, amigos) e a Universidade pela estrutura disponibilizada durante a realização desse trabalho. De maneira especial, queremos agradecer aos participantes do presente estudo pela disponibilidade, paciência e gentileza em responderem aos questionários quando empregados. Assim, nosso muito obrigado a todos que contribuíram direta e indiretamente para que essa pesquisa ocorresse. 


\section{Referências}

Almeida, E., \& Godoy, E. V. (2016). A evasão nos cursos de engenharia: uma análise a partir do COBENGE. http://www.abenge.org.br/cobe nge/arquivos/3/anais/anais/159848.pdf

Auerbach, R. P., Mortier, P., Bruffaerts, R., Alonso, J., Benjet, C., Cuijpers, P., \& Klessler. R. C. (2018). World Mental Health Surveys International College Student Project: Prevalence and distribution of mental disorders. J Abnorm Psychol. 127 (7), 623-638. 10.1037/ abn0000362

Auerbach, R. P., Alonso, J., Axinn, W. G., Cuijpers, P., Ebert, D. D., Green, J. G., \& Bruffaerts. R. (2016). Mental disorders among college students in the World Health Organization World Mental Health Surveys. Psychological Medicine, 46 (14), 2955-2970. 10.1017/S0033291716001665

Arnett, J. J. (2007). Emerging adulthood: What is it, and what is it good for? Child Development Perspectives, 1(2), 68-73. doi.org/10.1111/j.17508606.2007.00016.x

Bardagi, M., \& Hutz, C. S. (2005). Evasão universitária e serviços de apoio ao estudante: Uma breve revisão da literatura brasileira. Psicologia Revista. São Paulo, 14(2): 279-301. https://revistas.pucsp.br/index.php/psicorevista/article/view/18107/13463

Bohry, S. (2007). Crise Psicológica do Universitário e Trancamento Geral de Matrícula por motivos de Saúde. (Dissertação de mestrado) Universidade de Brasília, Brasília.

Carvalho, E. A., Bertolini, S. M. M. G., Milani, R. G., \& Martins, M. C. (2015). Índice de ansiedade em universitários ingressantes e concluintes de uma instituição de Ensino Superior. Ciência, Cuidado e Saúde, 14(3), 1290-1298. 10.4025/cienccuidsaude.v14i3.23594

Certo, A. C. T. (2016). Qualidade do sono e suas implicações ao nível da ansiedade depressão e stress nos estudantes do ensino superior. Relatório de Estágio para a obtenção de Grau de Mestre em Enfermagem Comunitária. Escola Superior de Saúde. Bragança.

Cosme, P. C., \& Durante, D. G. (2017). Estudar e trabalhar: impactos na formação acadêmica em secretariado executivo. Revista Expectativa, 16 (17), 44-65.

Costa, S. F. P. (2011). Dificuldades de Aprendizagem no Contexto Psicopedagógico. Profissão Docente, Uberaba, 20 (23), $154-157$.

Costa, E. F. O., Andrade, T. M., Neto. A. M. S., Melo, E. V., Rosa, A. C. A., Alencar, M. A., \& Silva, A. M. (2010). Common mental disorders among medical students at Universidade Federal de Sergipe: a cross-sectional study. Revista Brasileira de Psiquiatria, 32(1):11-9. 10.1590/S151644462010000100005

Daniel, W. W. (1995). Biostatistics: A foundation for analysis in the health sciences. United States: Jonh Wiley\& Sons.

Del Duca, G. F. D. Nahas, M. V. Hallal, P. C., \& Peres, K. G. (2014). Atividades físicas no lazer entre adultos de Florianópolis, Santa Catarina, Brasil: estudo populacional sobre as características das práticas e de seus praticantes. Ciência \& saúde coletiva. 19 (11), 4595-4604. 10.1590/1413-812320141911.16732013

Eisenberg, D., Gollust, S. E., Golberstein, E., \& Hefner, J. L. (2007). Prevalence and Correlates of Depression, Anxiety and Suicidality Among University Students. American Journal of Orthopsychiatry, 77(4), 534-532. 10.1037/0002-9432.77.4.534

Facundes, V. L. D., \& Ludermir, A. B. (2005). Common mental disorders among health care students. Revista Brasileira de Psiquiatria. 27(3). 10.1590/S1516-44462005000300007

Fiedler, P. T. (2008). Avaliação da qualidade de vida do estudante de medicina e da influência exercida pela formação acadêmica (Tese de doutorado). Universidade de São Paulo, São Paulo, SP. https://www.teses.usp.br/teses/disponiveis/5/5137/tde-10072008-161825/publico/PatriciaTempskiFiedler.pdf

Freitas, B. A. Costa, E. C. A. C., \& Costa, C. P. (2017). Fatores da evasão discente no curso de Engenharia Civil da Universidade Estadual da Paraíba. Revista Principia. 34, 69-76. Disponível em file:///C:/Users/Cliente/Downloads/1340-3621-1-PB.pdf

Gomes, L. F., \& Calixto, M. F. (2019). Saúde mental e ensino superior: a relação da universidade com o surgimento elou agravação de sofrimento mental em estudantes universitários. 2019. Trabalho de Conclusão de Curso (Bacharel em Enfermagem) Faculdade CESMAC do Sertão, AL. https://ri.cesmac.edu.br/bitstream/tede/561/1/Sa\%c3\%bade\%20mental\%20e\%20ensino\%20superio\%20-

$\% 20$ a $\% 20$ rela $\%$ c3\%a7\%c3\%a3o\%20da\%20universidade $\% 20$ com $\% 20$ o $\% 20$ surgimento $\% 20$ eou $\% 20$ agravo $\% 20$ de $\% 20$ sofrimento $\% 20$ mental $\% 20$ em $\% 20$ estud antes\%20universit\%c3\%a1rios..pdf

Grether, E. O. Becker, M. C. Menezes, H. M., \& Nunes. C. R. O. (2019). Prevalência de Transtornos Mentais Comuns entre Estudantes de Medicina da Universidade Regional de Blumenau (SC). Revista brasileira de educação médica. 43 (1), 276-285. 10.1590/1981-5271v43suplemento1-20180260

Gondim, S. M. G. (2002). Perfil profissional e mercado de trabalho: relação com a formação acadêmica pela perspectiva de estudantes universitários. Estudos de Psicologia. 7 (2), 299-309.10.1590/S1413-294X2002000200011

Harding, T.W. De Arango, M. V. Baltazar, J. Climent, C. E. Ibrahim, H. H., \& Ladrido-Ignacio L. (1980). Mental disorders in primary health care: a study of their frequency and diagnosis in four developing countries. Psychological Medicine, 10(2):231- 41. 10.1017/s0033291700043993

Holley, J. Crone, D. Tyson, P., \& Lovell, G. (2011). The effects of physical activity on psychological well-being for those with schizophrenia: a systematic review., British Journal of Clinical Psychology 50, 84-105. 10.1348/014466510X496220

Hosmer, D.W. And Lemeshow, S. (2000). Applied Logisic Regression. (2a ed.).

Ibrahim, A. K., Kelly, S.J., Adams, C. E., \& Glazebrook, C. (2013). A systematic review of studies of depression prevalence in university students. Journal of Psychiatric Research, 47, 391- 400. 10.1016/j.jpsychires.2012.11.015

Junior, M. A. G. N., Braga, Y. A., Marques, T. G., Silva, R. T., Vieira, S.D., Coelho, V. A. F., \& Regazzoni, L. A. A. (2015). Depressão em estudantes de Medicina. Revista Médica Minas Gerais, 25(4), 562-567. 10.5935/2238-3182.20150123 
Lima, J. K. A., \& Brito, A. P. A. (2020). Transtorno mental comum em estudantes de medicina de duas instituições de Salvador/Ba. In: JORNADA UNIFACS DE INICIAÇÃO CIENTÍFICA. 43 2020. Anais da 17 Jornada UNIFACS de Iniciação Científica- JUIC. Salvador: Universidade Salvador - UNIFACS. p. 1-4. https://www.unifacs.br/wp-content/uploads/2015/02/17-JUIC_2020_Jamily-Kaliny-Azevedo-Lima.pdf

Lincoln, A. K. Shepherd, A. Johnson, P. L., \& Castaneda-Sceppa, C. (2011). The impact of resistance exercise training on the mental health of older Puerto Rican adults with type 2 diabetes. The Journals of Gerontology, Series B: Psychological Sciences and Social Sciences, 66(5), 567-570. 10.1093/geronb/gbr034

Leão, A. M. Gomes, I. P. Ferreira, M. J. M., \& Cavalcanti, L. P. G. (2018). Prevalência e fatores associados à depressão e ansiedade entre estudantes universitários da área da saúde de um grande centro urbano do nordeste do Brasil. Revista Brasileira de Educação Médica, 42 (4), 55-65. 10.1590/1981$52712015 v 42 n 4 R B 20180092$

Ludermir, A. B. Melo Filho, D. A. (2002). Living conditions and occupational organization associated with common mental disorders. Revista de Saúde Pública. 36 (2), 213-21. 10.1590/S0034-89102002000200014

Ludermir, A. B., \& Lewis, G. (2005). Investigating the effect of demographic and socioeconomic variables on misclassification by the SRQ-20 compared with a psychiatric interview. Social Psychiatry and Psychiatr Epidemiology, 40(1):36-41. 10.1007/s00127-005-0840-2

Logan, J., Hughes, T., \& Logan, B. (2016). Overworked? An observation of the relationship between student employment and academic performance. Journal of College Student Retention: Research, Theory and Practice, 18, 250-262. 10.1177\%2F1521025115622777

Manimaran, S. Jayakumar, S. \& Lakshmi, K. B. (2016). An education management information system with simultaneous monitoring of stress stimulators for students Mental Health management. Technology and health care, Zurique, 24(6), 889-897. 10.3233/ THC-161250

Marin-Leon, L. Oliveira, H. B. Barros, M. B. A. Dalgalarrondo P., \& Botega, N. J. (2007). Social inequality and common mental disorders. Revista Brasileira de Psiquiatria. 29(3), 250-3. 10.1590/S1516-44462006005000060

Mari, J. J. \& Williams, P. (1985). A comparison of the validity of two psychiatric screening questionnaires (GHQ-12 and SRQ-20) in Brazil, Relative Operating Characteristic (ROC) analysis. Psychol Medicine, 15(3), 651-659. 10.1017/s0033291700031500

Mari, J. J., \& Williams, P. (1986). A validity study of a psychiatric screening questionnaire (SRQ20) in primary care in the city of Sao Paulo. British Journal of Psychiatry, 148:23-6. 10.1192/bjp.148.1.23

Mojtabai, R. Stuart, E. A. Hwang, I. Eaton, W. W. Sampson, N., \& Kessler, R. C. (2015). Long-term effects of mental disorders on educational attainment in the National Comorbidity Survey ten-year follow-up. Social Psychiatry and Psychiatric Epidemiology, 50(10), 1577-1591. 10.1007/s00127-015-1083-5

Moreira, J. K. P. Bandeira, M. Cardoso, C. S., \& Scalon, J. D. (2011). Prevalence of common mental disorders in the population attended by the Family Health Program. Jornal brasileiro de Psiquiatria. 60(3). 10.1590/S0047-20852011000300012

OPAS - Organização Pan-Americana Da Saúde. (2019). Organização Mundial da Saúde. Transtornos Mentais. Folha informativa. 2019. https://www.who.int/news-room/fact-sheets/detail/mental-disorders

Oikawa, F. M. (2019). Implicações do contexto universitário na saúde mental dos estudantes. 2019. Dissertação (Mestrado em Educação) Universidade Federal de São Carlos, Campus Sorocaba. Sorocaba.

Ogushi, M. M. P., \& Bardagi, M. P. (2015). Reflexões sobre a relação estudante- -universidade a partir de uma experiência de atendimento em orientação profissional. Extensio: Revista Eletrônica de Extensão, 12(19), 33-50. doi:10.5007/1807--0221.2015v12n19p33

Pereira, G. A. Capanema, H. X. M. Silva, M. M. Q. Garcia, I. L., \& Petroianu, A. (2015). Prevalência de Síndromes Funcionais em Estudantes e Residentes de Medicina. Revista Brasileira de Educação Médica, 39(3), 395-400. 10.1590/1981-52712015v39n3e00022014.

Perini, J. P. Delanogare, E., \& Souza, S. A. (2019). Transtornos mentais comuns e aspectos psicossociais em universitários do sul do Brasil. Revista de Ciências da Saúde. 31(1), 44-51. 10.14295/vittalle.v31i1.8678

Polydoro, S. A. J. Primi, R. Serpa, M. N. F. Zaroni, M. M. H., \& Pombal, K. C. P. (2001). Desenvolvimento de uma escala de integração ao ensino superior. Psico-USF. 6 (1), 11-7. 10.1590/S1413-82712001000100003

Quintana, A. M. Rodrigues, A.T. Goi, C. M. D., \& Bassi, L. A. (2004). Humanização e estresse na formação médica. Revista da Amrigs. 48 (1), $27-31$

Rego, C. C. A. B., and Rocha, N. M. F. (2009). Avaliando a educação emocional: subsídios para um repensar da sala de aula. Ensaio: Avaliação e Políticas. Púbicas em Educação., Rio de Janeiro, 17 (62), 135- 152. 10.1590/S0104-40362009000100007

Sanches, S. H. B., Osório, F. L., Udina., Santos, R. M., Crippa, J. A. S. (2012). Associação entre ansiedade e hipermobilidade articular: uma revisão sistemática. Revista Brasileira de Psiquiatria, 34 (11):53-68

Sampson, J. P. McClain, M - C. Musch, E., \& Reardon, R. C. (2013). Variables Affecting Readiness to Benefit From Career Interventions. The Career Development Quarterly, 61(2), 98-109. 10.1002/j.2161-0045.2013.00040.x

Santos, L. S. Ribeiro, Í. J. S. Boery, E. N., \& Boery, R. N. S. O. (2017). Qualidade de vida e transtornos mentais comuns em estudantes de medicina. Cogitare Enfermagem. 22(4), 1-7. 10.5380/ce.v22i4.52126

Silva, A. O., \& Neto, C. J. P. (2014). Associação entre níveis de atividade física e transtorno mental comum em estudantes universitários. Motricidade, 10(1), 49-59. 10.6063/motricidade.10(1).2125

Silva, P.L.B. C. Silva, B. F. F. Chagas, K. K. A. C. R. Tortola, M. B. A. Caldeira, R; L. R. (2019). Transtorno Mental comum entre Estudantes de Enfermagem e Fatores Envolvidos. Revista de Enfermagem do Centro Oeste Mineiro. 9, 1-7. http://dx.doi.org/10.19175/recom.v9i0.3191 
Research, Society and Development, v. 10, n. 3, e48910313585, 2021

(CC BY 4.0) | ISSN 2525-3409 | DOI: http://dx.doi.org/10.33448/rsd-v10i3.13585

Silva, M. Z., \& Andrade, A. L. (2016). Avaliando pensamentos negativos sobre a carreira: o desenvolvimento de uma medida (EPNC). Revista Brasileira de Orientação Profissional. 17 (2). http://pepsic.bvsalud.org/scielo.php?script=sci_arttext\&pid=S1679-33902016000200006

Soares, J. F., \& Collares, A. C. M. (2006). Recursos familiares e o desempenho cognitivo dos alunos do ensino básico brasileiro. Revista de Ciências Sociais. 49 (3), 615-650. 10.1590/S0011-52582006000300007

Tosevski, D. L., Milovancevic, M. P., \& Gajic, S. D. (2010). Personality and psychopathology of university students. Current Opinion in Psychiatric, 23(1), 48-52. 10.1097/YCO.0b013e328333d625

UFS - Universidade Federal de Sergipe. (2019). Anuário Estatístico da UFS: 2016 - 2018 / org. OLIVEIRA, Kleber Fernandes de. SANTOS, Rosalvo Ferreira. São Cristóvão: COPAC/PROPLAN. 202 p. http://indicadores.ufs.br/uploads/page_attach/path/7127/Anu_rio_Estat_stico_da_UFS_2016-20172018_final.pdf

UFS - Universidade Federal de Sergipe. (2018). Anuário Estatístico da UFS: 2015 - 2017 / org. OLIVEIRA, Kleber Fernandes de. SANTOS, Rosalvo Ferreira. São Cristóvão: COPAC/PROPLAN. 190 p. http://indicadores.ufs.br/uploads/page_attach/path/5878/Anu_rio_Estat_stico_da_UFS_2015-20162017_v1_m_dia_qualidade.pdf

UFS - Universidade Federal de Sergipe. Conselho Universitário. (2007). Resolução Nº 02/2007/CONEP, de 13 de março de 2007. São Cristóvão - SE, Brasil

UFS - Universidade Federal de Sergipe. Conselho Universitário. (2009). Resolução nº 174/2009/CONEPE, de 18 de dezembro de 2009. São Cristóvão - SE, Brasil

Wipfli, B. Landers, D. Nagoshi, C., \& Ringenbach, S. (2011). An examination of serotonin and psychological variables in the relationship between exercise and mental health. Scandinavian Journal of Medicine \& Science in Sports, 21, 474-481. 10.1111/j.1600-0838.2009.01049.x 